\title{
RUNX2 promotes hepatocellular carcinoma cell migration and invasion by upregulating MMP9 expression
}

\author{
QIAN WANG ${ }^{1}$, WEI YU ${ }^{1}$, TAO HUANG ${ }^{1}$, YAN $\mathrm{HU}^{2}$ and $\mathrm{CHANGSHAN} \mathrm{HUANG}{ }^{1}$ \\ ${ }^{1}$ Department of Hepato-Biliary-Pancreatic Surgery, The Affiliated Cancer Hospital of Zhengzhou University; \\ ${ }^{2}$ Department of Medical Oncology, People's Hospital of Henan Province, Zhengzhou, Henan 450000, P.R. China
}

Received April 21, 2016; Accepted August 23, 2016

DOI: 10.3892/or.2016.5101

\begin{abstract}
Runt-related transcription factor 2 (RUNX2) was first identified as a transcription factor to play an important role in different biological processes of osteoblast and chondrocyte, including differentiation and migration. Recently, RUNX2 has been implicated in promigratory/proinvasive behavior in different human malignancies. In the present study, we demonstrated that the RUNX2 mRNA and protein expression were both increased significantly in HCC tissues and cell lines. High RUNX2 expression was correlated obviously with poor clinicopathological characteristics including multiple tumor nodes, high histological grading, venous infiltration and advanced tumor-node-metastasis (TNM) stage. In addition, we demonstrated that RUNX2 was a prognostic indicator for predicting 5-year overall survival and disease-free survival of HCC patients. Our studies showed that RUXN2 overexpression promoted, while RUNX2 knockdown inhibited HCC cell migration and invasion in vitro. Notably, RUNX2 positively regulated matrix metalloproteinase 9 (MMP9) accumulation in HCC cells. Furthermore, we confirmed that RUNX2 was positively correlated with MMP9 expression in HCC tissues by Pearson correlation analysis. Mechanistically, we demonstrated that MMP9 overexpression increased HCC cell migration and invasion, while MMP9 knockdown reduced $\mathrm{HCC}$ cell migration and invasion in vitro. Alteration of MMP9 expression partially abrogated the effects of RUNX2 on HCC cell migration and invasion, which suggests that RUNX2 developed its pro-metastatic biological function by upregulating the expression of MMP9 in HCC cells. In conclusion, our results reveal that RUNX2 promotes HCC cell migration and invasion by MMP9-mediated pathway, and potentially serves as a new prognostic biomarker and in therapeutic strategies for HCC.
\end{abstract}

Correspondence to: Professor Changshan Huang, Department of Hepato-Biliary-Pancreatic Surgery, The Affiliated Cancer Hospital of Zhengzhou University, 127 Dongming Road, Zhengzhou, Henan 450000, P.R. China

E-mail: huangcs1234@sina.com

Key words: RUNX2, hepatocellular carcinoma, migration, invasion, matrix metalloproteinase 9

\section{Introduction}

Hepatocellular carcinoma (HCC) is one of the most common cancers worldwide and the third leading cause of cancerrelated death globally (1). Despite considerable advances in treatment modalities, the long-term survival of HCC patients remains poor because of its high recurrence and early metastasis (2). However, the underlying mechanism responsible for the development and progression of $\mathrm{HCC}$ has not been fully elucidated (3). Therefore, it is urgent to develop a novel therapeutic target involved in HCC.

Runt-related transcription factor 2 (RUNX2), which belongs to RUNX family, are distinctive by a highly conserved 128 amino acid DNA binding/protein-protein interaction domain (4). It functions as a major regulator for osteoblast differentiation and regulates endochondral bone formation physiological progression (5-7). Recent studies suggested that RUNX2 are involved in many types of human cancer development, progression and metastasis (8-10). In vitro studies showed that RUNX2 promoted the migration and invasion capacity of prostate cancer cells $(11,12)$. In breast cancer, RUNX2 promoted tumorsphere formation by regulating soluble E-cadherin expression associated with the TAZ transcriptional co-activator (13). Moreover, it was recently reported that RUNX2 played a critical role in the process of epithelial to mesenchymal transition (EMT) whose characteristics include increased migration, invasion and metastasis potential by upregulating the transcription factors, such as SOX9 and SMAD3 (12). In addition, RUNX2 was essential for cellular movement and cytoskeleton remodeling. In non-small cell lung cancer, the increased RUNX2 presents resistance to cisplatin chemosensitivity. Furthermore, RUNX2 promoted migration and invasion potential of thyroid tumor cells by activating the expression levels of MMPs (14). These studies suggest that RUNX2 probably functions as an oncogene for tumorigenesis and metastasis. However, the precise function of RUNX2 and the underlying mechanisms in HCC remain unclear.

In the present study, we demonstrated that RUNX2 expression is upregulated in HCC. Clinical analysis reveals that the increased RUNX2 expression was associated with poor prognostic features and was an independent prognostic marker for predicting survival of HCC patients. RUNX2 promotes cell migration and invasion by regulating MMP9 expression in HCC cells. Mechanistically, the pro-metastatic effect of 
RUNX2 could be abrogated by inhibiting MMP9 expression in vitro. Our data suggest that RUNX2 probably promotes MMP9 expression and thus, induces the metastasis of HCC cells.

\section{Materials and methods}

Clinical tissues and data. Ninety-six HCC tissues and matched tumor-adjacent tissues were obtained from patients including 82 males and 14 females, who underwent curative resection surgery in the Department of Hepato-Biliary-Pancreatic Surgery, The Affiliated Cancer Hospital of Zhengzhou University from January 2006 to December 2009. All tissues were used after obtaining informed consent. Patients did not receive preoperative chemotherapy or embolization. The demographic and clinicopathological data were obtained through medical records. The experimental protocols were approved by the Zhengzhou University Ethics Committee according to the Declaration of Helsinki.

Cell lines and transfection. The human $\mathrm{HCC}$ cell lines (HepG2, MHCC-97L, Hep3B, SMMC-7721, MHCC-97H and HCCLM3) and immortalized normal hepatic cell line LO2 were purchased from the Institute of Biochemistry and Cell Biology (Chinese Academy of Sciences, Shanghai, China). The cells were cultured in complete Dulbecco's modified Eagle's medium (DMEM; Invitrogen, Carlsbad, CA, USA) supplemented with $10 \%$ fetal bovine serum (FBS; Gibco, Grand Island, NY, USA) at $37^{\circ} \mathrm{C}$ in a humidified incubator in $5 \% \mathrm{CO}_{2}$.

Retroviral vectors pMMP-RUNX2 and pMMP-MMP9 were generated by inserting the cDNA into pMMP. The specific siRNA against RUNX2 (5'-UAACAGCAGAGGCAUUUCG UAGCUC-3'), MMP9 (5'-CUAUGGUCCUCGCCCUGAA-3') and scramble siRNA (5'-UUCUCCGAACGUGUCACGUUU GUGC-3') were synthesized by Shanghai GenePharma Co., Ltd. (Shanghai,China). Cells were transfected with the siRNAs mentioned above using Lipofectamine 2000 (Invitrogen) according to the manufacturer's instructions.

Real-time quantitative reverse transcription polymerase chain reaction $(q R T-P C R)$. Total RNA was extracted from respective HCC cells and clinical samples using TRIzol reagent (Invitrogen). The first strand cDNA was reverse transcribed with miRNA assay kit (Applied Biosystems, Foster City, CA, USA) and quantified by a SYBR ${ }^{\circledR}$ Premix Ex Taq ${ }^{\mathrm{TM}}$ II (Perfect Real-Time) kit (Takara Bio, Inc., Shiga, Japan) and performed on the ABI PRISM 7300 Sequence Detection System (Applied Biosystems). qPCR primer against RUNX2 (HQP016478) and GAPDH (HQP006940) were purchased from Genecopoeia (Guangzhou, China).

Western blot analysis. The HCC cells and clinical tissues were collected and lysed, then the protein concentration was quantified using the BCA reagent (Pierce, Rockford, IL, USA). A total of $30 \mu \mathrm{g}$ protein was separated by $10 \%$ SDS-PAGE and transferred onto a PVDF membrane (Bio-Rad Laboratories, Hercules, CA, USA). The membranes were probed with the following primary antibodies: RUNX2 (1:1,000; Cell Signaling Technology, Inc., Danvers, MA, USA), MMP9 (1:1,000; Santa
Cruz Biotechnology, Santa Cruz, CA, USA), GAPDH (G8140; US Biological, Salem, MA, USA) overnight. The membranes were incubated with appropriate HRP-conjugated secondary antibody (ZSGB-BIO, Beijing, China). Protein bands were visualized using an enhanced chemiluminescence kit (Amersham, Little Chalfont, UK).

Immunohistochemical staining. Samples were fixed in formalin and embedded in paraffin and sections were cut at $4 \mu \mathrm{m}$ thickness. RUNX2 and MMP9 (1:400; Cell Signaling Technology) antibody was performed in immunohistochemistry according to a standard streptavidin-peroxidase-conjugated (SP-IHC) procedures. The staining results for the RUNX2 and MMP9 protein were semi-quantitatively evaluated by the staining intensity and the percentage of positive staining cells. The percentage of positive cells was divided into four grades: 0 for $<5 \%$; 1 for $6-25 \%$; 2 for $26-50 \% ; 3$ for $51-75 \%$ and 4 for $>75 \%$. Staining intensity was assessed by four degrees: 0 , negative; 1 , weak; 2 , moderate; and 3, strong. Each section was assayed for ten independent high magnification $(\mathrm{x} 400)$ fields to get the average scores.

Cell migration and invasion assays. Transwell cell migration and invasion assays were carried out by the $8 \mu \mathrm{M}$ pore-sized Transwell inserts (Nalge Nunc International Corp., Naperville, IL, USA). Transfected cells were seeded at $2.5 \times 10^{5} / \mathrm{ml}$ in $200 \mu 1$ serum-free DMEM medium into the upper chamber, and $750 \mu \mathrm{l}$ DMEM medium containing 10\% FBS was placed in the lower chamber. After 24-h incubation, cells were fixed in $4 \%$ paraformaldehyde for $20 \mathrm{~min}$ and stained with $0.1 \%$ crystal violet dye for $15 \mathrm{~min}$. The cells on the inner layer were softly removed with a cotton swab; 1:6 dilution Matrigel invasion chamber (BD Biosciences, San Jose, CA, USA) was performed for invasion assays and the following assays were same as before.

Statistical analysis. Results are shown as mean \pm standard deviation. The SPSS 13 (SPSS, Inc., Chicago, IL, USA) and GraphPad Prism 5 software (GraphPad Software, Inc., San Diego, CA, USA) were used for Pearson Chi-squared test and the multivariate Cox regression analysis. Two-tailed Student's t-test, a Kaplan-Meier plot, a log-rank test, a Spearman's rank correlation coefficient or an ANOVA were used to evaluate the statistical significance. Difference was defined as $\mathrm{P}<0.05$.

\section{Results}

The expression level of RUNX2 in HCC tissues and cells. We investigated the expression level of RUNX2 in 96 pairs of HCC tissues and corresponding adjacent non-tumor tissues by qRT-PCR and western blot analysis. We found that RUNX2 mRNA and protein expression in HCC tissues were both obviously higher than those in corresponding adjacent non-tumor tissues ( $\mathrm{P}<0.05$; Fig. 1A and B). Moreover, RUNX2 mRNA levels were increased in HCC cell lines (HepG2, Hep3B, SMMC-7721, MHCC-97L, HCCLM3 and MHCC-97H) compared to the normal hepatocyte cell line, $\mathrm{LO} 2(\mathrm{P}<0.05$; Fig. 1C). In addition, the levels of RUNX2 protein showed the same result with mRNA levels in all cell lines by western blot analysis ( $\mathrm{P}<0.05$; Fig. 1D). Furthermore, RUNX2 expres- 

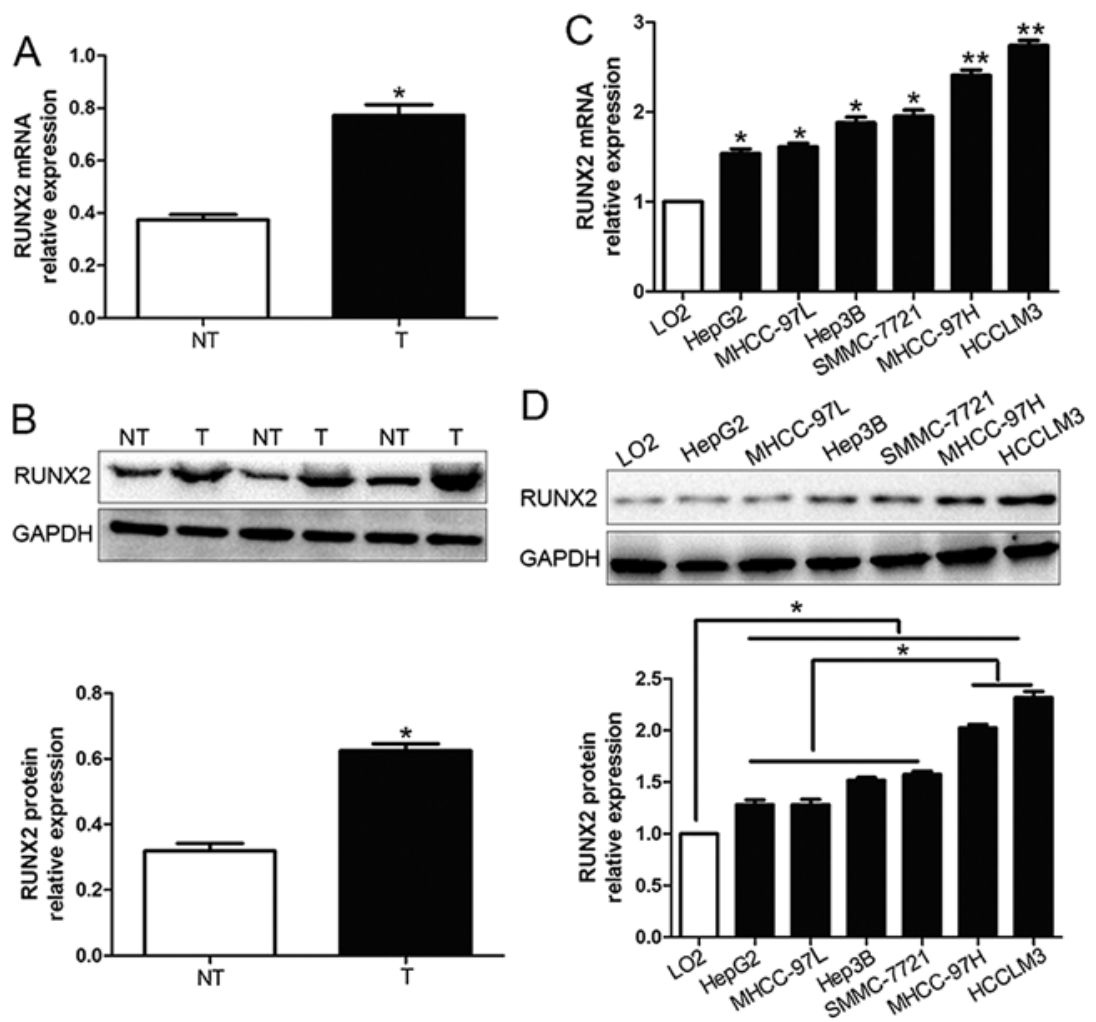

Figure 1. RUNX2 is highly expressed in HCC tissues and cell lines. (A) Relative RUNX2 mRNA expression levels in tumor tissues (T) and matched adjacent non-tumor tissues (NT) were determined by qRT-PCR $(\mathrm{n}=96)$. (B) Representative western blot analysis of RUNX2 expression in the HCC (T) and non-tumor tissues (NT) was shown; "P $<0.05$ by t-test. Comparing differences in the expression level of RUNX2 mRNA (C) and protein (D) between HCC cell lines with different metastatic potentials and the immortalized hepatic cell line LO2. $\mathrm{n}=$ three repeats with similar results; ${ }^{*} \mathrm{P}<0.05$ by ANOVA. ${ }^{* *} \mathrm{P}<0.01$.
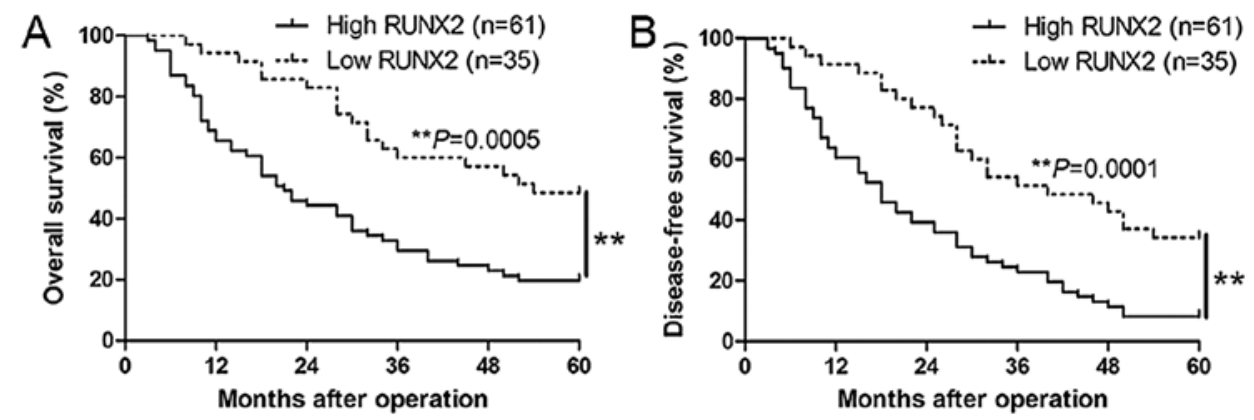

Figure 2. Prognostic significance of RUNX2 in HCC cases. (A) Kaplan-Meier 5-year overall and (B) disease-free survival curves of HCC patients according to the level of RUNX2 protein expression. The RUNX2 high expression group $(\mathrm{n}=61)$; RUNX2 low expression group $(\mathrm{n}=35)$. The mean expression value obtained for RUNX2 of the 96 HCC samples detected by western blot analysis was chosen as the cut-off value. ${ }^{* *} \mathrm{P}<0.01$ by log-rank test.

sion in MHCC-97H and HCCLM3, which was considered as highly metastasis were prominently higher than those in the low metastasis HCC cell lines (HepG2, Hep3B, SMMC-7721 and MHCC-97L) $(\mathrm{P}<0.05$; Fig. 1D). Thus, these results suggest that elevated RUNX2 expression probably plays a critical role in the development of HCC.

Correlation between RUNX2 expression and clinicopathological features. We defined the mean level of RUNX2 protein as a cut-off value to distinguish the RUNX2 expression level. As shown in Table I, the high expression of RUNX2 protein was significantly associated with multiple tumor nodes $(\mathrm{P}=0.006)$, venous infiltration $(\mathrm{P}=0.009)$, high Edmondson-Steiner grading $(\mathrm{P}=0.001)$ and advanced tumor-node-metastasis $(\mathrm{TNM})$ stage
$(\mathrm{P}=0.004)$. Hence, these data suggest that the increased expression of RUNX2 is correlated with adverse prognostic features of HCC. In addition, Kaplan-Meier analysis revealed that high RUNX2 expression was correlated with worse overall survival $(\mathrm{P}=0.0005$; Fig. 2A) and disease-free survival $(\mathrm{P}=0.0001$, Fig. 2B) of HCC patients. In addition, RUNX2 expression was a novel independent factor for predicting both 5-year overall and disease-free survival in $\mathrm{HCC}$ patients $(\mathrm{P}=0.021$ and 0.039 , respectively, Table II). Taken together, these results highlight the potential value of RUNX2 for the outcome of HCC.

RUNX2 promotes HCC cell migration and invasion. To explore the biological function of RUNX2 in HCC, we transfected HCC cell line MHCC-97L with empty vector (EV) or RUNX2 
Table I. Clinical correlation of RUNX2 expression in HCC $(\mathrm{n}=96)$.

\begin{tabular}{|c|c|c|c|c|}
\hline \multirow[b]{2}{*}{$\begin{array}{l}\text { Clinical } \\
\text { parameters }\end{array}$} & \multirow[b]{2}{*}{$\begin{array}{c}\text { Cases } \\
\text { (n) }\end{array}$} & \multicolumn{2}{|c|}{ Expression level } & \multirow[b]{2}{*}{$\begin{array}{c}\text { P-value } \\
\left({ }^{\mathrm{a}} \mathrm{P}<0.05\right)\end{array}$} \\
\hline & & $\begin{array}{c}\text { RUNX2 }^{\text {high }} \\
(\mathrm{n}=61)\end{array}$ & $\begin{array}{c}\text { RUNX2 }^{\text {low }} \\
(\mathrm{n}=35)\end{array}$ & \\
\hline
\end{tabular}

\begin{tabular}{lllll}
\hline Age (years) & & & & \\
$<65$ & 28 & 18 & 10 & 0.923 \\
$\geq 65$ & 68 & 43 & 25 &
\end{tabular}

Gender

$\begin{array}{lrrr}\text { Male } & 82 & 52 & 30 \\ \text { Female } & 14 & 9 & 5 \\ \text { Tumor size }(\mathrm{cm}) & & & \\ <5 & 37 & 27 & 10 \\ \geq 5 & 59 & 34 & 25\end{array}$

Tumor number

\begin{tabular}{lrrrr} 
Solitary & 76 & 43 & 33 & \\
Multiple & 20 & 18 & 2 & \\
Edmondson & & & & $0.001^{\text {a }}$ \\
I+II & 32 & 13 & 19 & \\
III+IV & 64 & 48 & 16 & \\
TNM stage & & & & $0.004^{\text {a }}$ \\
I+II & 65 & 35 & 30 & \\
III+IV & 31 & 26 & 5 & \\
Venous infiltration & & & & $0.009^{\text {a }}$ \\
Present & 26 & 22 & 4 & \\
Absent & 70 & 39 & 31 & \\
AFP (ng/ml) & & & & 0.619 \\
$<400$ & 38 & 23 & 15 & \\
$\geq 400$ & 58 & 38 & 20 & \\
HBsAg & & & & 0.810 \\
Positive & 84 & 53 & 31 & \\
Negative & 12 & 8 & 4 & \\
\hline
\end{tabular}

retroviruses $(\mathrm{P}<0.01$; Fig. $3 \mathrm{~A})$. We demonstrated that RUNX2 overexpression prominently promoted $\mathrm{HCC}$ cell migration and invasion in MHCC-97L ( $\mathrm{P}<0.05$; Fig. 3B). Moreover, RUNX2 was knocked down using a specific siRNA in HCCLM3 cells (P<0.05; Fig. 3C). As expected, downregulated RUNX2 led to a significant reduction of cell migration and invasion $(\mathrm{P}<0.05$; Fig. 3D). These data suggest that RUNX2 regulates the migration and invasion of HCC cells.

RUNX2 positively regulates MMP9 in HCC. The positive correlation between RUNX2 and MMP9 expression was reported previously in breast cancer (15-17), thus, we evaluated the effect of RUNX2 on MMP9. Amazingly, our results showed that RUNX2 overexpression obviously increased the expression level of MMP9 in MHCC-97L cells $(\mathrm{P}<0.05$; Fig. 4A). Moreover, RUNX2 knockdown significantly decreased the level of MMP9 in HCCLM3 cells ( $\mathrm{P}<0.05$; Fig. 4B). We then analysed RUNX2 and MMP9 expression by immunostaining in HCC samples. Both RUNX2 and MMP9 expression in cancer tissues were significantly higher than those in paired non-cancerous tissues $(\mathrm{P}<0.05$; Fig. $4 \mathrm{C})$. Moreover, IHC scores were evaluated for semi-quantitative analysis, we found a strong positive correlation between RUNX2 and MMP9 $(r=0.534, P<0.001$; Fig. 4D). These data revealed that RUNX2 exerts its biological promotive function through elevating expression of MMP9.

RUNX2 promotes $H C C$ cell migration and invasion by increasing MMP9. To explore whether MMP9 participates in RUNX2 mediated promotion of HCC cell migration and invasion, MMP9 expression was significantly increased by MMP9 retroviruses in MHCC-97L cells $(\mathrm{P}<0.05$; Fig. 5A) and significantly knocked down using a specific siRNA in HCCLM3 cells $(\mathrm{P}<0.05$; Fig. 5C). As shown in Fig. 5B and 5D, MMP9 overexpression obviously promoted, while MMP9 knockdown obviously blocked HCC cell migration and invasion $(\mathrm{P}<0.05$; respectively). In addition, RUNX2 overexpressing MHCC-97L cells were subsequently transfected with MMP9 siRNA $(\mathrm{P}<0.05$; Fig. 6A). MMP9 knockdown markedly abrogated the function of exogenous RUNX2 overexpression, causing a significant decrease in the number of migrated and invaded cells $(\mathrm{P}<0.05$; Fig. 6B). Similarly, MMP9 overexpression promoted HCC cell migration and invasion in RUNX2knockdown HCCLM3 cells $(\mathrm{P}<0.05$, respectively; Fig. 6C and D). In conclusion, these results suggest that MMP9 may function as a downstream factor in RUNX2 mediated promotion of HCC cell migration and invasion.

\section{Discussion}

In the present study, we initially checked RUNX2 expression level in 96 specimens of HCC tissues. Our results show that the RUNX2 expression level in HCC was obviously higher than that in non-tumor tissues. Increased expression levels of RUNX2 mRNA and protein were also confirmed in different HCC cell lines, especially in the highly metastatic cell lines. Moreover, elevated expression of RUNX2 was significantly correlated with multiple tumor nodes, high histological grade, TNM stage and venous infiltration. These data suggest that the increased RUNX2 expression is correlated with adverse prognostic characters in HCC. Furthermore, patients with higher RUNX2 had a worse prognosis of HCC patients, which was consistent with that in acute lymphoblastic leukemia patients (18). Multivariate Cox repression analysis demonstrated that RUNX2 was a novel independent prognostic factor for predicting survival of HCC patients. Taken together, these results indicate that RUNX2 expression is critical for prognosis of HCC patients.

Previous studies suggest that RUNX2 functions as an oncogene in breast cancer development through Wnt and TGF- $\beta$ signaling pathways (19-21). Moreover, downregulated RUNX2 inhibits the invasion of osteosarcoma (22). In the present study, we found that RUNX2 overexpression prominently promoted MHCC-97L cell migration and invasion and RUNX2 knockdown significantly reduced the migrated and invaded HCCLM3 cells. These data suggest that RUNX2 exerts 
Table II. Multivariate Cox regression analysis of 5-year overall and disease-free survival of 96 HCC patients.

\begin{tabular}{|c|c|c|c|c|c|c|}
\hline \multirow[b]{2}{*}{ Variables } & \multicolumn{3}{|c|}{ Overall survival } & \multicolumn{3}{|c|}{ Disease-free survival } \\
\hline & HR & $95 \% \mathrm{CI}$ & P-value & HR & $95 \% \mathrm{CI}$ & P-value \\
\hline RUNX2 & 2.179 & $1.107-4.658$ & $0.021^{\mathrm{a}}$ & 3.648 & $1.084-5.103$ & $0.039^{\mathrm{a}}$ \\
\hline Edmondson grade & 2.148 & $0.846-5.082$ & 0.068 & 1.981 & $0.834-4.596$ & 0.135 \\
\hline TNM stage & 1.134 & $1.013-1.948$ & $0.023^{\mathrm{a}}$ & 1.023 & $1.004-1.768$ & $0.003^{\mathrm{a}}$ \\
\hline No. of tumor nodule & 1.712 & $0.684-4.249$ & 0.214 & 1.132 & $0.954-3.821$ & 0.873 \\
\hline Venous infiltration & 1.078 & $0.987-1.247$ & 0.057 & 1.023 & $0.658-1.784$ & 0.856 \\
\hline
\end{tabular}

HR, hazard ratio; CI, confidence interval; astatistically significant.

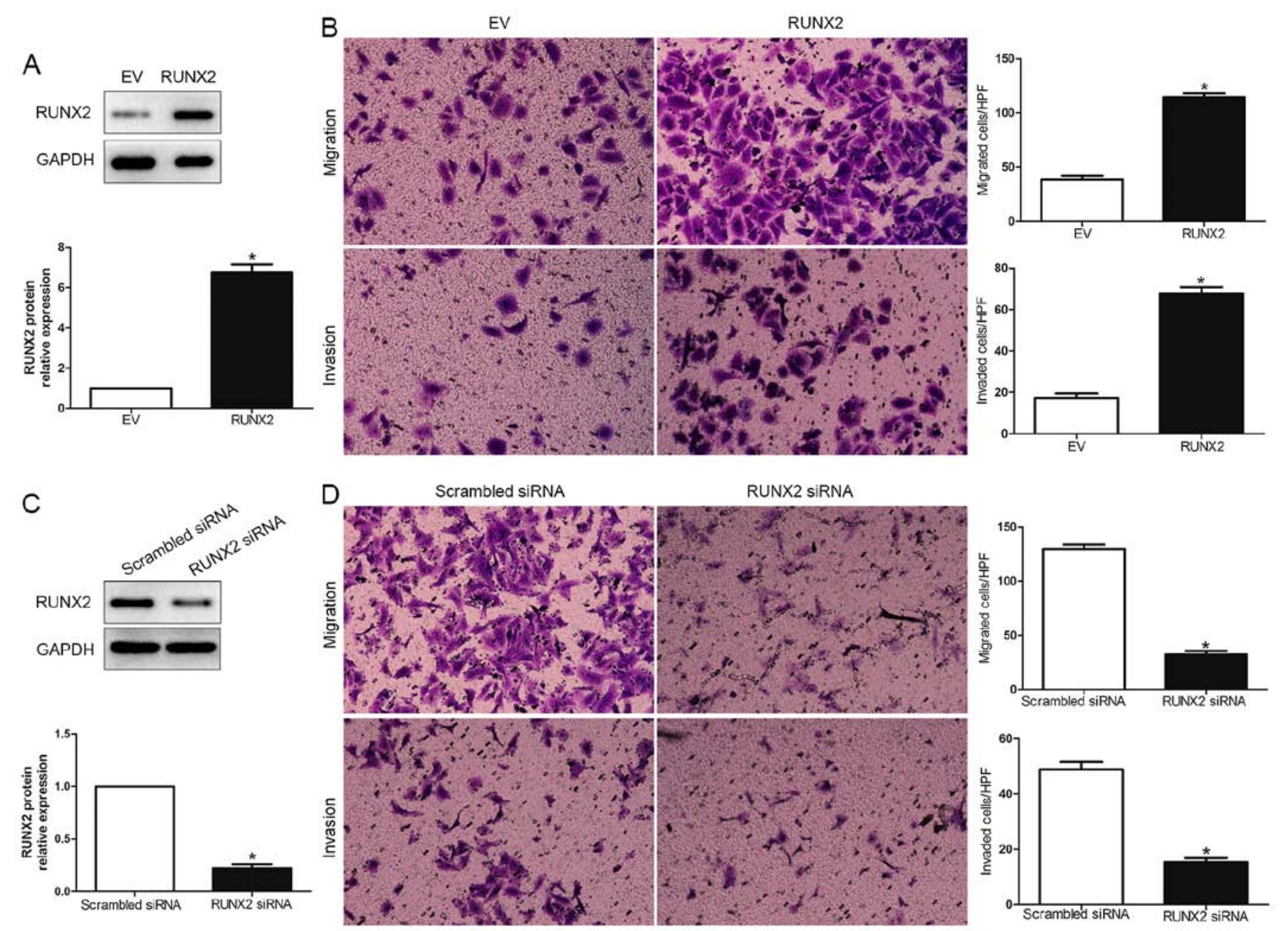

Figure 3. RUNX2 promotes HCC cell migration and invasion. (A) MHCC-97L cells that were transfected with empty vector (EV) or RUNX2 retroviruses were subjected to western blot analysis for RUNX2. $\mathrm{n}=$ six independent experiments; ${ }^{*} \mathrm{P}<0.05$ by t-test. (B) Cell migration and invasion as measured by Transwell assays were increased by RUNX2 overexpression in MHCC-97L cells as compared with control cells. $\mathrm{n}=$ three repeats with similar results, ${ }^{*} \mathrm{P}<0.05$ by $\mathrm{t}$-test. HPF, high power field. (C) HCCLM3 cells that were transfected with scrambled siRNA or RUNX2 siRNA were subjected to western blot for RUNX2. $\mathrm{n}=$ six independent experiments; " $\mathrm{P}<0.05$ by t-test. (D) RUNX2 knockdown HCCLM3 cells conferred a less number of migrated and invaded cells as compared with control cells. $\mathrm{n}=$ three repeats with similar results; ${ }^{*} \mathrm{P}<0.05$ by t-test. HPF, high power field.

its biological function on HCC cell migration and invasion. Peng et al (23) reported that RUNX2 promoted trophoblast invasion via MMP9 expression. Our data demonstrated that RUNX2 overexpression upregulated MMP9 in MHCC-97L cells and RUNX2 knockdown led to MMP9 reduction in
HCCLM3 cells. In addition, we demonstrated that the MMP9 expression level in high RUNX2-expressed HCC tissues was prominently higher than that from low RUNX2-expressed group. Spearman correlation analysis suggest a positive correlation between RUNX2 and MMP9 expression in HCC 

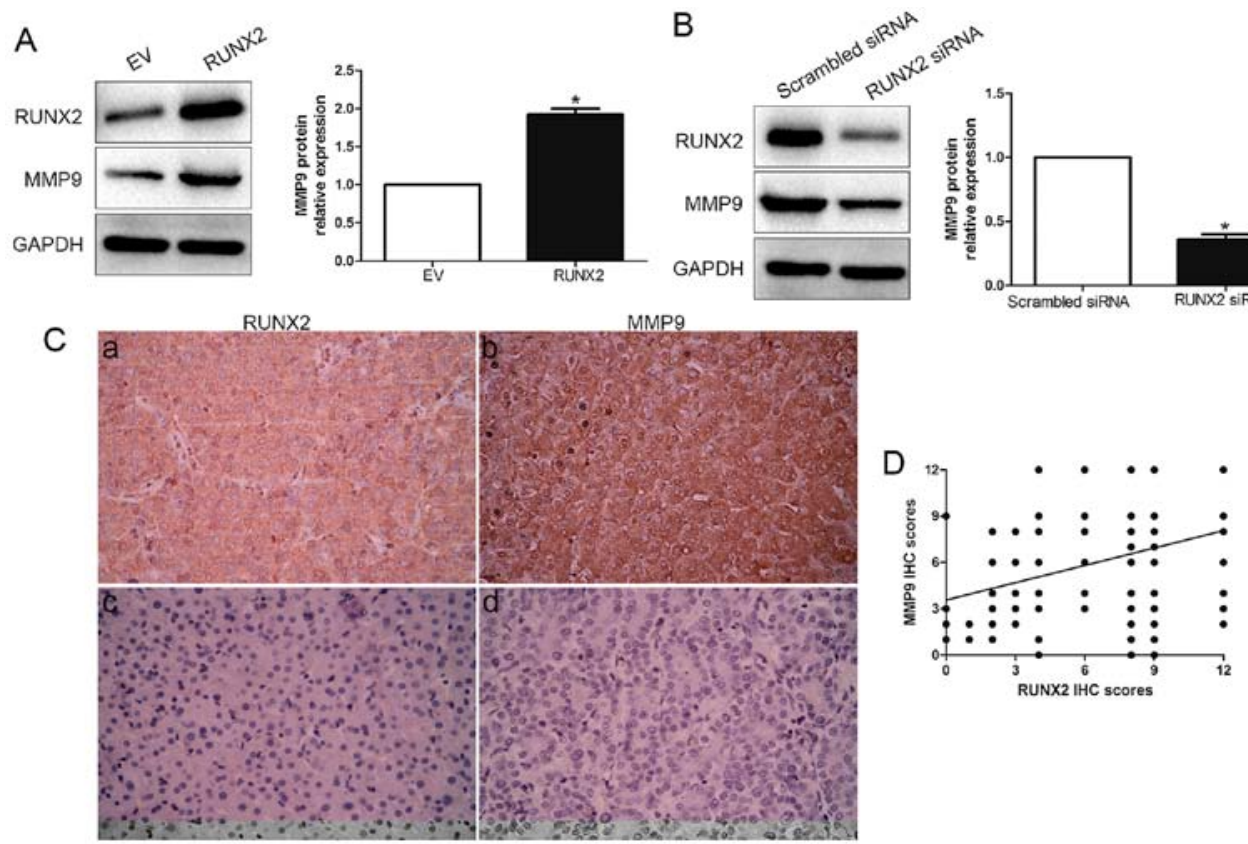

Figure 4. RUNX2 regulates MMP9 abundance in HCC. (A) RUNX2 overexpression increased the level of MMP9 protein in MHCC-97L cells. $n=$ six independent experiments; ${ }^{*} \mathrm{P}<0.05$ by t-test. (B) RUNX2 knockdown inhibited the expression of MMP9 protein in HCCLM3 cells. $\mathrm{n}=$ six independent experiments; ${ }^{*} \mathrm{P}<0.05$ by t-test. (C) In cases of high RUNX2 protein expression (a), there was strong MMP9 protein expression (b) in the same tissue section. In contrast, in the case of low RUNX2 protein expression (c), there was no detectable MMP9 protein expression (d). (D) RUNX2 and MMP9 expression in 96 HCC samples analyzed by immunohistochemistry. A significant positive correlation between RUNX2 and MMP9 expression was observed in HCC.
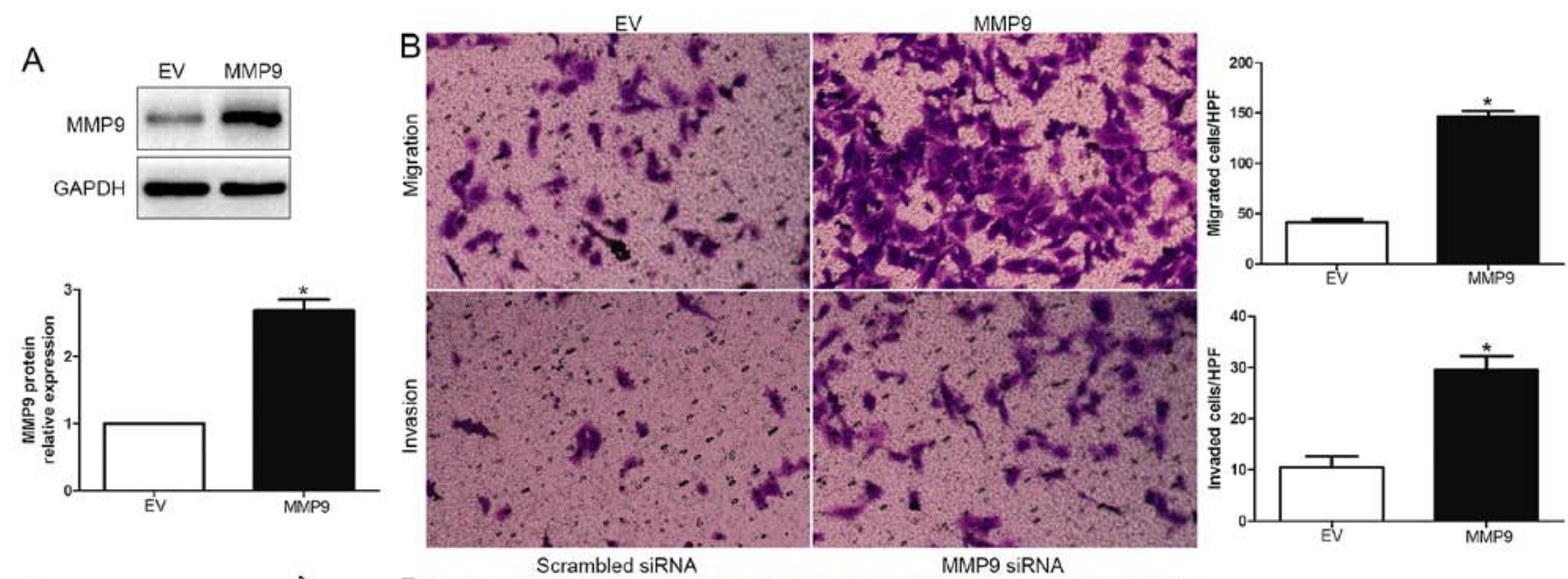

C
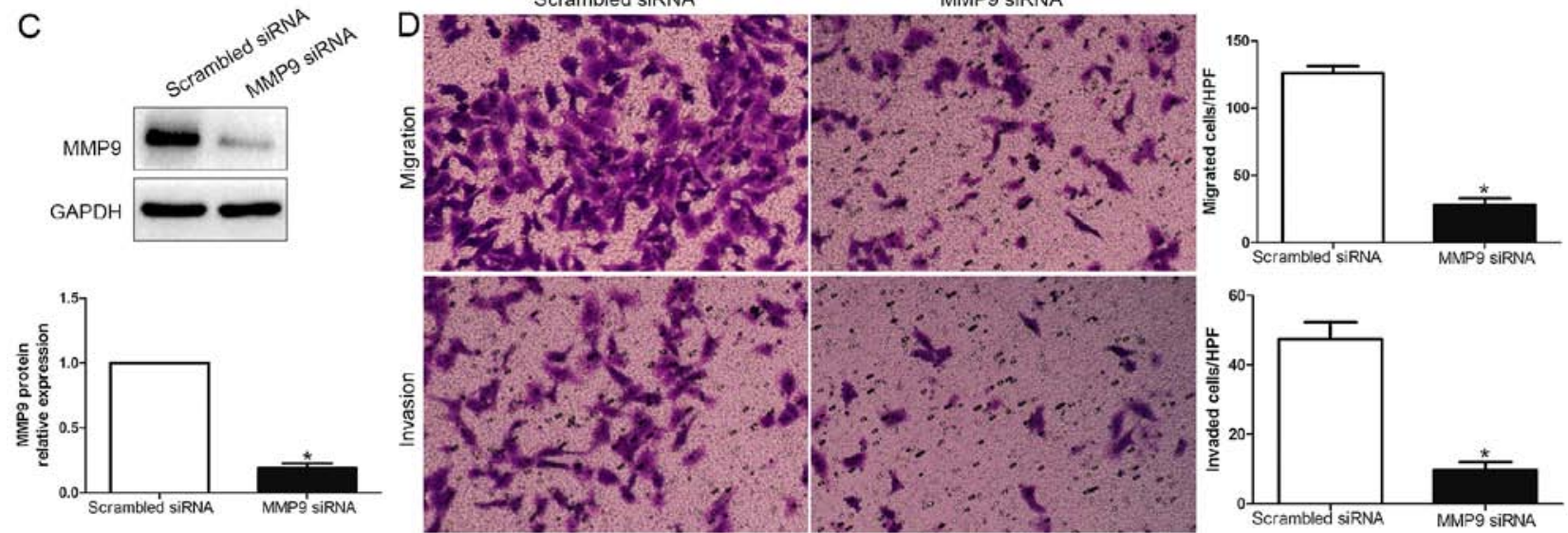

Figure 5. RUNX2 exerts pro-metastatic effect by promoting MMP9 expression. (A) MHCC-97L cells that were transfected with empty vector (EV) or MMP9 retroviruses were subjected to western blot analysis for MMP9. $\mathrm{n}=$ six independent experiments; ${ }^{*} \mathrm{P}<0.05$ by $\mathrm{t}$-test. (B) Cell migration and invasion as measured by Transwell assays were increased by MMP9 overexpression in MHCC-97L cells as compared with control cells. $\mathrm{n}=$ three repeats with similar results, ${ }^{*} \mathrm{P}<0.05$ by t-test. HPF, high power field. (C) HCCLM3 cells that were transfected with scrambled siRNA or MMP9 siRNA were subjected to western blot analysis for MMP9. $\mathrm{n}=$ six independent experiments; ${ }^{*} \mathrm{P}<0.05$ by $\mathrm{t}$-test. (D) MMP9 knockdown HCCLM3 cells conferred a less number of migrated and invaded cells as compared with control cells. $\mathrm{n}=$ three repeats with similar results; ${ }^{\mathrm{P}} \mathrm{P}<0.05$ by $\mathrm{t}$-test. $\mathrm{HPF}$, high power field. 
A
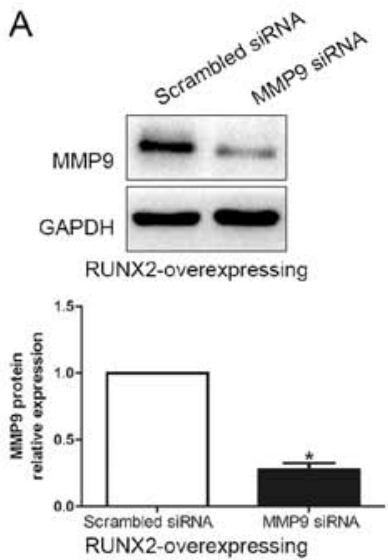

C
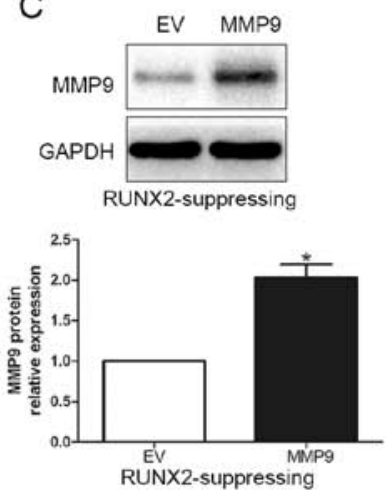

B Scrambled siRNA
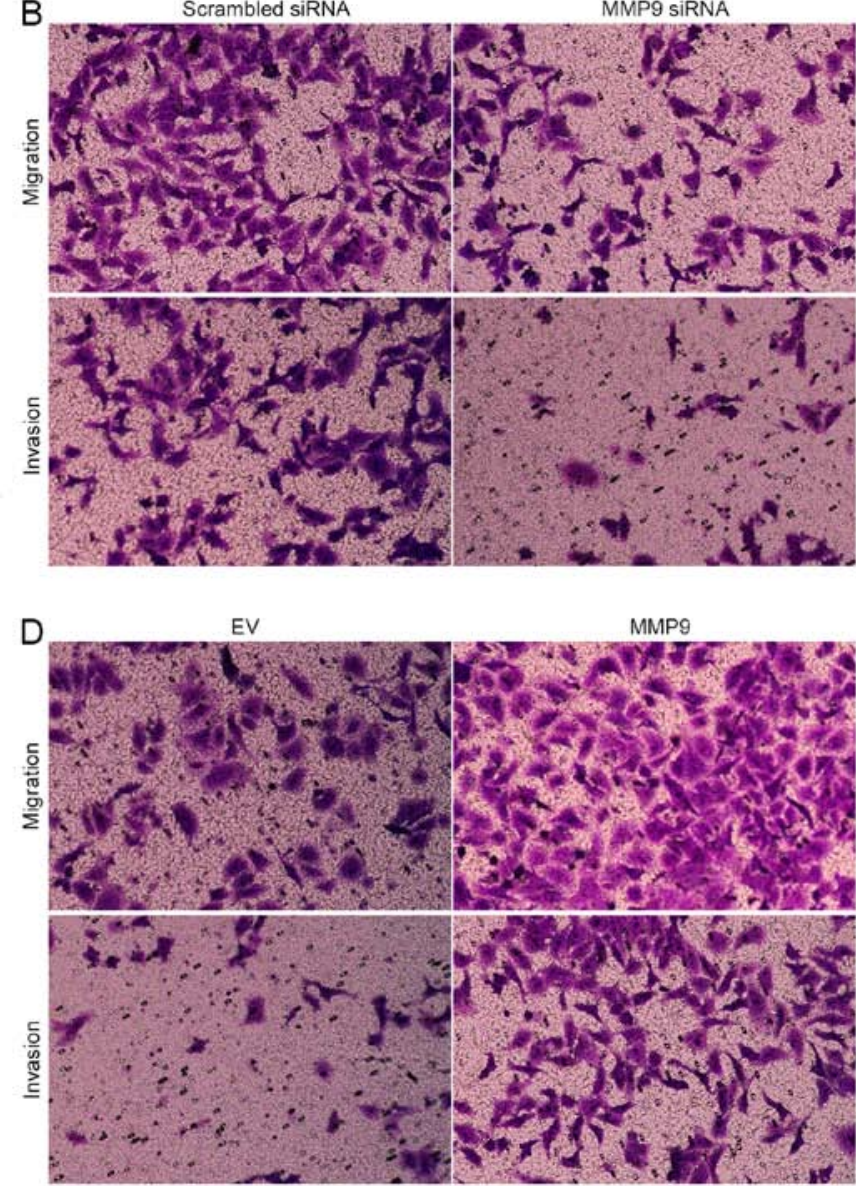
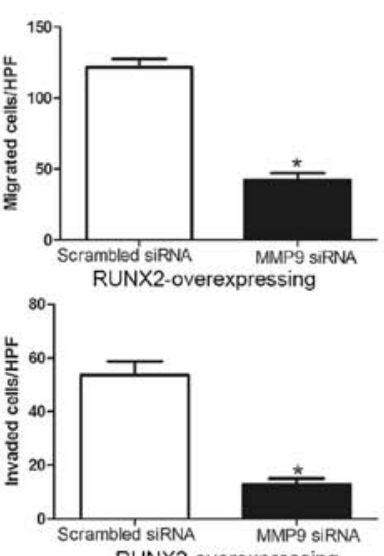

RUNX2-overexpressing
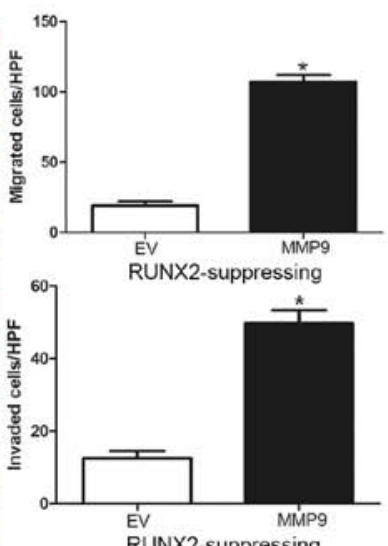

Figure 6. Alterations of MMP9 levels influence the effect of RUNX2 on HCC cells. (A) RUNX2-overexpressing MHCC-97L cells that were transfected with scrambled siRNA or MMP9 siRNA were subjected to immunoblotting for MMP9. $\mathrm{n}=$ six independent experiments; ${ }^{2} \mathrm{P}<0.05$ by $\mathrm{t}$-test. (B) MMP9 knockdown decreased migration and invasion in RUNX2-overexpressing MHCC-97L cells. $\mathrm{n}=$ three repeats with similar results, ${ }^{\mathrm{P}}<0.05$ by t-test. $(\mathrm{C})$ RUNX2-suppressing HCCLM3 cells that were transfected with empty vector (EV) or MMP9 retroviruses were subjected to immunoblotting for MMP9. $\mathrm{n}=$ six independent experiments; ${ }^{*} \mathrm{P}<0.05$ by t-test. (D) Restoring MMP9 expression increased migration and invasion in RUNX2-suppressing HCCLM3 cells. $\mathrm{n}=$ three repeats with similar results, ${ }^{*} \mathrm{P}<0.05$ by t-test.

tissues. Taken together, these data indicate that RUNX2 positively regulate MMP9 accumulation in HCC. Furthermore, we confirmed that MMP9 overexpression obviously promoted HCC cell migration and invasion in MHCC-97L cells, while MMP9 knockdown obviously inhibited migration and invasion in HCCLM3 cells, which was consistent with previous reports. Moreover, the promotion in HCC migration and invasion by RUNX2 overexpression could be abolished by MMP9 knockdown, and the suppression in HCC cell migration and invasion by RUNX2 knockdown could be reverted by restoring MMP9 expression. Taken together, these data indicate that RUNX2 promotes HCC cell migration and invasion by increasing MMP9 expression. In osteoblast, RUNX2 exerts as a regulator in metastasis by interaction with PI3K/AKT signaling $(24,25)$. Similarly, in breast cancer, RUNX2 directly regulates the expression of MMP9 and MMP13. Furthermore, RUNX2 overexpression upregulates transcription factors (SOX9, SMAD3 and SNAI2) implicated in the process of epithelial to mesenchymal transition (12,26-29). Therefore, we need further investigation to explore the molecular mechanisms between RUNX2 and MMP9 in HCC.

In conclusion, the data show that the expression of RUNX2 is elevated in HCC tissues and cell lines and its high expres- sion is associated with malignant clinicopathological features. We confirm that RUNX2 is an independent prognostic marker for predicting 5-year survival of HCC patients. We demonstrate that RUNX2 promotes HCC cell migration and invasion in vitro. Mechanistically, we suggest that RUNX2 may promote HCC invasion and metastasis by increasing MMP9. Taken together, we consider that RUNX2 may potentially act as a clinical biomarker, and may also be a therapeutic target in $\mathrm{HCC}$.

\section{Acknowledgements}

The present study was supported by a grant from the Scientific Research Foundation of Henan (no. 092102310090).

\section{References}

1. El-Serag HB and Rudolph KL: Hepatocellular carcinoma: Epidemiology and molecular carcinogenesis. Gastroenterology 132: 2557-2576, 2007.

2. Talwalkar JA and Gores GJ: Diagnosis and staging of hepatocellular carcinoma. Gastroenterology 127 (Suppl 1): S126-S132, 2004.

3. Jemal A, Bray F, Center MM, Ferlay J, Ward E and Forman D: Global cancer statistics. CA Cancer J Clin 61: 69-90, 2011. 
4. Ito Y: Oncogenic potential of the RUNX gene family: 'overview'. Oncogene 23: 4198-4208, 2004.

5. Li J, Hao L, Wu J, Zhang J and Su J: Linarin promotes osteogenic differentiation by activating the BMP-2/RUNX2 pathway via protein kinase A signaling. Int J Mol Med 37: 901-910, 2016.

6. Ozaki T, Nakamura M and Shimozato O: Novel implications of DNA damage response in drug resistance of malignant cancers obtained from the functional interaction between $\mathrm{p} 53$ family and RUNX2. Biomolecules 5: 2854-2876, 2015.

7. Sugimoto H, Nakamura M, Yoda H, Hiraoka K, Shinohara K, Sang M, Fujiwara K, Shimozato O, Nagase H and Ozaki T: Silencing of RUNX2 enhances gemcitabine sensitivity of p53-deficient human pancreatic cancer AsPC-1 cells through the stimulation of TAp63-mediated cell death. Cell Death Dis 6: e1914, 2015.

8. Wysokinski D, Blasiak J and Pawlowska E: Role of RUNX2 in breast carcinogenesis. Int J Mol Sci 16: 20969-20993, 2015.

9. Shrivats AR, McDermott MC, Klimak M, Averick SE, Pan H, Matyjaszewski K, Mishina Y and Hollinger JO: Nanogelmediated RNAi against Runx 2 and Osx inhibits osteogenic differentiation in constitutively active BMPR1A osteoblasts. ACS Biomater Sci Eng 1: 1139-1150, 2015.

10. Fujita T, Azuma Y, Fukuyama R, Hattori Y, Yoshida C, Koida M, Ogita K and Komori T: Runx2 induces osteoblast and chondrocyte differentiation and enhances their migration by coupling with PI3K-Akt signaling. J Cell Biol 166: 85-95, 2004.

11. Akech J, Wixted JJ, Bedard K, van der Deen M, Hussain S, Guise TA, van Wijnen AJ, Stein JL, Languino LR, Altieri DC, et al: Runx 2 association with progression of prostate cancer in patients: Mechanisms mediating bone osteolysis and osteoblastic metastatic lesions. Oncogene 29: 811-821, 2010.

12. Baniwal SK, Khalid O, Gabet Y, Shah RR, Purcell DJ, Mav D, Kohn-Gabet AE, Shi Y, Coetzee GA and Frenkel B: Runx2 transcriptome of prostate cancer cells: Insights into invasiveness and bone metastasis. Mol Cancer 9: 258, 2010.

13. Brusgard JL, Choe M, Chumsri S, Renoud K, MacKerell AD Jr, Sudol M and Passaniti A: RUNX2 and TAZ-dependent signaling pathways regulate soluble E-cadherin levels and tumorsphere formation in breast cancer cells. Oncotarget 6: 28132-28150, 2015.

14. Sancisi V, Borettini G, Maramotti S, Ragazzi M, Tamagnini I, Nicoli D, Piana S and Ciarrocchi A: Runx2 isoform I controls a panel of proinvasive genes driving aggressiveness of papillary thyroid carcinomas. J Clin Endocrinol Metab 97: E2006-E2015, 2012.

15. Mendoza-Villanueva D, Deng W, Lopez-Camacho C and Shore P: The Runx transcriptional co-activator, CBFbeta, is essential for invasion of breast cancer cells. Mol Cancer 9: 171, 2010.

16. Pratap J, Javed A, Languino LR, van Wijnen AJ, Stein JL, Stein GS and Lian JB: The Runx 2 osteogenic transcription factor regulates matrix metalloproteinase 9 in bone metastatic cancer cells and controls cell invasion. Mol Cell Biol 25: 8581-8591, 2005 .
17. Selvamurugan N, Kwok S and Partridge NC: Smad3 interacts with JunB and Cbfa1/Runx2 for transforming growth factor-beta1stimulated collagenase-3 expression in human breast cancer cells. J Biol Chem 279: 27764-27773, 2004.

18. Edwards H, Xie C, LaFiura KM, Dombkowski AA, Buck SA, Boerner JL, Taub JW, Matherly LH and Ge Y: RUNX1 regulates phosphoinositide 3-kinase/AKT pathway: Role in chemotherapy sensitivity in acute megakaryocytic leukemia. Blood 114: 2744-2752, 2009.

19. Tandon M, Chen Z, Othman AH and Pratap J: Role of Runx2 in IGF-1R $\beta /$ Akt- and AMPK/Erk-dependent growth, survival and sensitivity towards metformin in breast cancer bone metastasis. Oncogene: Jan 25, 2016 Epub ahead of print. doi: 10.1038/ onc. 2015.518

20. Sancisi V, Gandolfi G, Ragazzi M, Nicoli D, Tamagnini I, Piana S and Ciarrocchi A: Cadherin 6 is a new RUNX2 target in TGF- $\beta$ signalling pathway. PLoS One 8: e75489, 2013.

21. van der Deen M, Akech J, Wang T, FitzGerald TJ, Altieri DC, Languino LR, Lian JB, van Wijnen AJ, Stein JL and Stein GS: The cancer-related Runx 2 protein enhances cell growth and responses to androgen and TGFbeta in prostate cancer cells. J Cell Biochem 109: 828-837, 2010.

22. Zeng $\mathrm{H}$ and $\mathrm{Xu} \mathrm{X}$ : RUNX2 RNA interference inhibits the invasion of osteosarcoma. Oncol Lett 9: 2455-2458, 2015.

23. Peng B, Zhu H, Klausen C, Ma L, Wang YL and Leung PC: GnRH regulates trophoblast invasion via RUNX2-mediated MMP2/9 expression. Mol Hum Reprod 22: 119-129, 2016.

24. Cohen-Solal KA, Boregowda RK and Lasfar A: RUNX2 and the $\mathrm{PI} 3 \mathrm{~K} / \mathrm{AKT}$ axis reciprocal activation as a driving force for tumor progression. Mol Cancer 14: 137, 2015.

25. Tandon M, Chen $\mathrm{Z}$ and Pratap J: Runx2 activates PI3K/Akt signaling via mTORC2 regulation in invasive breast cancer cells. Breast Cancer Res 16: R16, 2014.

26. Niu DF, Kondo T, Nakazawa T, Oishi N, Kawasaki T, Mochizuki K, Yamane T and Katoh R: Transcription factor Runx2 is a regulator of epithelial-mesenchymal transition and invasion in thyroid carcinomas. Lab Invest 92: 1181-1190, 2012.

27. Zhang X, Akech J, Browne G, Russell S, Wixted JJ, Stein JL, Stein GS and Lian JB: Runx2-Smad signaling impacts the progression of tumor-induced bone disease. Int J Cancer 136: 1321-1332, 2015.

28. Chimge NO, Baniwal SK, Little GH, Chen YB, Kahn M, Tripathy D, Borok Z and Frenkel B: Regulation of breast cancer metastasis by Runx 2 and estrogen signaling: The role of SNAI2. Breast Cancer Res 13: R127, 2011.

29. Zaidi SK, Sullivan AJ, van Wijnen AJ, Stein JL, Stein GS and Lian JB: Integration of Runx and Smad regulatory signals at transcriptionally active subnuclear sites. Proc Natl Acad Sci USA 99: 8048-8053, 2002. 